\title{
The development of adjunctive drinking by rats: Conditioned and unconditioned components
}

\author{
J. D. KEEHN and EMOKE STOYANOV \\ York University, Toronto, Ontario, Canada
}

\begin{abstract}
We exposed 8 food-deprived male white rats to 20 experimental sessions with food reinforcement scheduled at fixed 60-sec intervals, and measured eat-drink latency, lick-bout duration, and local lick rate, as well as total water intake in a comparison with drinking under continuous reinforcement. The animals developed polydipsia over these sessions by taking increasingly frequent drinks with decreasing latencies as experience with the reinforcement schedule mounted. Local lick rate, however, did not change. The durations of these adjunctive drinks was also unchanged in average magnitude, but they became more stereotyped (less variable) from initial to terminal performance. We concluded that adjunctive drinking is conditionable in terms of frequency, latency, and variability in drink-bout duration, but not with respect to the local rate of licking or the average magnitude of lick-bout duration. This implies that distinct timing and volume regulation mechanisms are involved in the development of adjunctive drinking.
\end{abstract}

Schedule-induced polydipsia is supernormal water intake that results from an eat-drink behavioral sequence adopted by rats exposed to intermittent responsedependent or response-independent food schedules (Falk, 1961; Segal, Oden, \& Deadwyler, 1965). Along with schedule-induced aggression, it is classified as an adjunctive behavior because it relates lawfully to reinforcement schedule parameters without being required for reinforcement (Falk, 1972; Looney \& Cohen, 1982), but adjunctive aggression is identified behaviorally (pecking or biting a target) and not by its effect on the target, whereas it is the reverse with adjunctive drinking. In this case, its effect (polydipsia), rather than its behavioral dimensions (see Gilbert, 1958), is the normal identifying mark of adjunctive drinking (Falk, 1981). Problems with this manner of identification have been elaborated by Roper (1981).

The behavioral status of adjunctive drinking is uncertain (Pear \& Eldridge, 1984; Wetherington, 1982), but the fact that it develops with experience is indisputable. Several recent studies have described that experience as Pavlovian conditioning (Millenson, Allen, \& Pinker, 1977; Plonsky, Driscoll, \& Rosellini, 1985; see also Porter \& Hamm, 1984). They show the development of stimulus control of total water consumption with experience, but, since none describe the dimensions of drinking by which this comes about, exactly what is conditioned remains unknown. The present report is an attempt to fill

Parts of the data were presented at the 93rd Annual Convention of the American Psychological Association, Los Angeles, August 1985. Financial support was provided by a Natural Science and Engineering Research Council Scholarship to E.S. and an Atkinson College Research Grant to J.D.K. We thank Jackie Logan for the preparation of the manuscript and an anonymous reviewer for invaluable suggestions.

The authors' mailing address is: Department of Psychology, York University, Atkinson College, $\mathbf{4 7 0 0}$ Keele St., North York, Ont., Canada M3J 2R7. this gap. In addition to total fluid intake in the development of schedule-induced polydipsia, we measured four dimensions of drinking that could contribute to this total: drink-bout frequency, drink-bout duration, eat-drink latency, and local rate of licking. These measures were also taken under a conventional and an extended baseline condition with continuous reinforcement. Studies of induced drinking that focus on its terminal excessiveness require only a measure of preexperimental water intake as a control (cf. Roper, 1981; Timberlake, 1982, for problems and alternatives), but, since we were concerned with development in several dimensions of drinking, we employed an additional extended baseline control in case such developments occurred in the absence of intermittent reinforcement.

Inasmuch as rats' licking occurs in bursts rather than as individual licks (Davis \& Keehn, 1959; Stellar \& Hill, 1952), we consider local lick rate and bout duration as topographical dimensions of drinking behavior. Although it is responsive to the level of deprivation, time of testing, and drinking spout size and accessibility (Cone, 1974; Hard, 1976), there is little variability in local licking rates of an individual rat under constant conditions (Corbit \& Luschei, 1969; Hard, 1976; Keehn \& Arnold, 1960), and the duration of a particular drinking bout can be viewed as analogous to response magnitude in Pavlovian conditioning. Thus, we take it as our starting point that local rate and bout duration are topographical specifications of individual drinks and that latency is a measure of response probability.

\section{METHOD}

\section{Subjects}

The subjects were 8 naive 90 -day-old male rats bred from local Wistar stock. They were housed in individual home cages with water freely available, and maintained at approximately $85 \%$ of their 90 day-old individual free-feeding weights. 


\section{Apparatus}

Experimental spaces were standard Grason-Stadler operant chambers mounted in ventilated chests. A graduated water-bottle was attached to the outside of the chamber door, with the outlet protruding through a hole in the door $5 \mathrm{~cm}$ above floor level and $16 \mathrm{~cm}$ from the food magazine. Water-bottle spouts were approximately $5 \mathrm{~mm}$ in diameter and contained a ball bearing to offset leakage. The spouts were angled into the chamber such that the lower lip was $2 \mathrm{~mm}$ inside the chamber, and each animal was always trained with the same spout in the same box at the same time of day. Spout contacts were routed via Grason-Stadler drinkometers to a microcomputer control system (Micro Interfaces), which also controlled the scheduling of reinforcers (45-mg regular Noyes pellets) and data printouts.

\section{Procedure}

The subjects were trained to barpress with a continuous reinforcement schedule in which every barpress was reinforced (continuous reinforcement). Barpressing was established in 1 session with all 8 subjects, after which the continuous reinforcement baseline schedule was maintained for 3 more sessions (normal volume control) with 4 rats (Group 1) and 15 sessions (as a control for pretreatment changes in drink dimensions) with the other 4 (Group 2). Each baseline session terminated $10 \mathrm{~min}$ after an animal secured 100 reinforcers. After this, reinforcement was scheduled on a fixed-interval 60-sec schedule (FI 60-sec) in which only the first barpress after the end of each minute was reinforced. Twenty experimental sessions with this schedule were run at the same time on successive days, each one terminating approximately $10 \mathrm{~min}$ after an animal had secured 100 reinforcers. Water intakes, times of reinforcement, and water spout contacts were recorded and printed out at the end of every session. To minimize effects of occasional nonlick tube contacts through posture shifting, sniffing, biting, and so forth, on local lick-rate measurement, we defined a drink bout as a burst of licks at least $3 \mathrm{sec}$ in duration. Likewise, a minimum criterion of $5 \mathrm{sec}$ with no tube contact served to differentiate one lick bout from another. This criterion excludes "noisy" data at the expense of genuine bursts of licking shorter than $3 \mathrm{sec}$ in duration.

\section{RESULTS}

The animals drank very little while securing 100 food pellets under continuous reinforcement. The modal number of drink bouts for all animals over all sessions was 2 , and these bouts occurred unsystematically with respect to the number of pellets eaten before a drink, the latency from pellet delivery to first lick, and the duration of a drink once licking began. The essentially random nature of such baseline drinking is illustrated for subject $M 1$ in Figure 1, which shows eat-drink latencies (upper) and drink-bout durations (lower) accumulated over all 15 baseline sessions of this animal. Records of baseline drink latencies and durations by the other animals are equally lacking in order. Additional drinks that occurred in the $10 \mathrm{~min}$ after reinforcement ceased were likewise erratic as to interbout interval and bout duration. Except for the first, latencies of those bouts have no meaning, because they follow after drinks, not a food pellet.

Once a bout of licking began, however, local lick rate was relatively invariant at close to 4 licks $/ \mathrm{sec}$. This rate persisted throughout baseline sessions, as shown in Table 1 . The table contains average means and standard deviations of local lick rates, eat-drink latencies, and
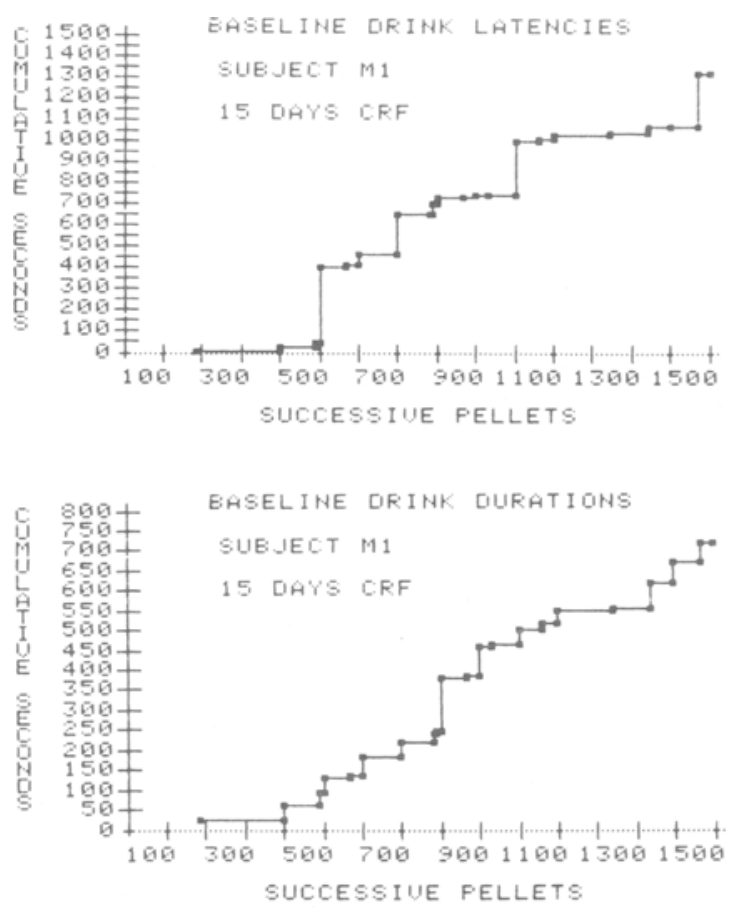

Figure 1. Drinks accumulated by a representative subject (M1) over the whole 15 sessions with the continuous reinforcement food schedule. Drinking by other subjects was similarly unsystematic.

drink-bout durations in those sessions in which all 4 animals in Group 2 took at least 2 drinks while securing 100 food pellets in a baseline session. There is no consistent effect of baseline session experience on either number of drinks, latency of drinks, duration of drinks, or local lick rate once drinking began.

There were no differences in session water intake between the animals exposed for 3 or 15 sessions of continuous reinforcement; over all sessions, Group 1 ( 3 sessions) and Group 2 (15 sessions) averaged $3.7 \mathrm{ml}(S D=$ $1.7)$ and $4.1 \mathrm{ml}(S D=2.1)$ water intake per session, respectively. Hence, both groups were combined for further data analysis. For the combined groups, Figure 2 shows the mean water intake (upper) and mean number of interpellet intervals (lower, $100 \mathrm{max}$ ) in which the

Table 1

Averaged Means and Averaged Standard Deviations of Local Lick Rates (Licks/Second), Drink Durations (Seconds), and Eat-Drink Latencies (Seconds) in Specific Sessions

\begin{tabular}{|c|c|c|c|c|c|c|c|}
\hline \multirow[b]{2}{*}{ Session } & \multirow[b]{2}{*}{$N$} & \multicolumn{2}{|c|}{ Duration } & \multicolumn{2}{|c|}{ Latency } & \multicolumn{2}{|c|}{ Local Rate } \\
\hline & & Mean & $S D$ & Mean & $S D$ & Mean & $S D$ \\
\hline 5 & 4 & 33.2 & 19.5 & 98.4 & 109.9 & 4.0 & 1.3 \\
\hline 9 & 4 & 58.5 & 47.6 & 13.7 & 8.6 & 4.2 & 1.6 \\
\hline 10 & 4 & 46.0 & 34.7 & 45.0 & 57.9 & 4.0 & 1.2 \\
\hline 11 & 4 & 43.6 & 32.5 & 18.4 & 12.2 & 4.1 & 1.7 \\
\hline 13 & 4 & 46.8 & 39.2 & 15.8 & 10.2 & 4.2 & 1.3 \\
\hline
\end{tabular}

Note-The above sessions were those in which all four animals in Group 2 took at least two drinks while securing 100 pellets in the extended baseline sessions with continuous reinforcement. The modal number of drink bouts within 100 pellets was 2 . 

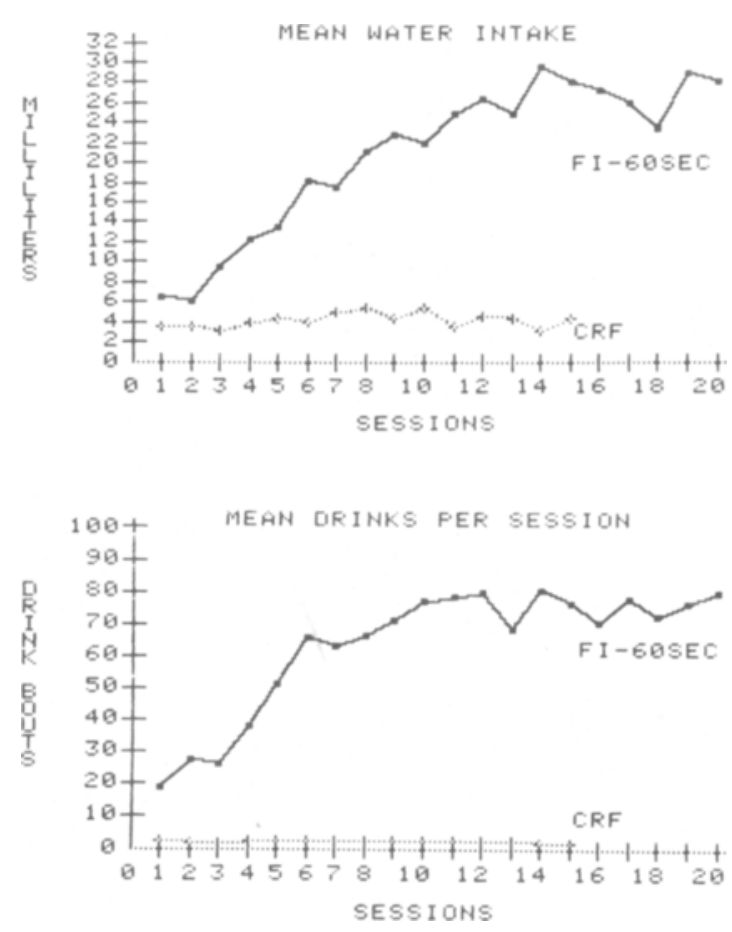

Figure 2. (Upper) Mean water intakes of all subjects under continuous (CRF) and intermittent (FI $60 \mathrm{sec}$ ) reinforcement. Under CRF, Sessions 1-3 are for Group 1 and Sessions 4-15 are for Group 2. (Lower) Mean drinks per sessions (Max 100) of all subjects under continuous (CRF) and intermittent (FI $60 \mathrm{sec}$ ) reinforcement. Under CRF, Sessions 1-3 are for Group 1 and Sessions 4-15 are for Group 2.

animals engaged in a bout of drinking while securing 100 pellets under the FI 60-sec reinforcement schedule. Comparable data for the sessions with continuous reinforcement are included in the figure for comparison. Analysis of variance by repeated measures revealed a significant sessions effect for both water intake $[F(7,19)=13.02$, $p<.0001]$ and mean drinks per session $[F(7,19)=$ $15.04, p<.0001]$. The mean number of intervals per session in which the animals drank stabilized by Session 12 , but mean water intake continued to rise for another 1 or 2 sessions. In almost all cases, only one drinking bout occurred in an interpellet interval.

As can be seen from Figure 3, neither the mean nor the standard deviation of local licking rate changed over the period in which drink frequency and volume of water intake increased. A comparison with Table 1, however, shows that local lick rates were consistently lower under the intermittent schedule than under the continuous reinforcement schedule. All means in all sessions shown in Figure 3 were less than 4 licks/sec, whereas in the comparable baseline sessions (Sessions 5, 9, 10, 11, and 13) local lick rates were 4 licks/sec or greater. A $t$ test for correlated means between each animal's lick rates averaged over Sessions 5, 9, 10,11, and 13 under the continuous and intermittent reinforcement conditions, however, was not significant for either local lick-rate means $[t(3)=1.21, p>.10]$ or standard deviations $[t(3)=2.24, p>.10]$.

Drink durations under the intermittent reinforcement schedule were considerably shorter than those under continuous reinforcement, where long drinks occurred after an average of 75 pellets had been consumed. Figure 4 (lower) shows that the mean of drink-bout durations did not change over experimental sessions. The standard deviations of drink-bout durations decreased, however, from a mean of $7.8 \mathrm{sec}(S D=1.6)$ over the first three sessions to a mean of $4.4 \mathrm{sec}(S D=1.1)$ across Sessions 10 through 12. By $t$ test for correlated means, this differ-

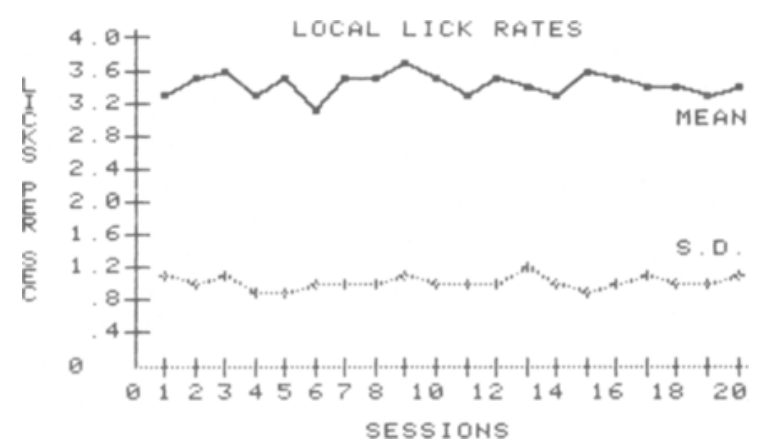

Figure 3. Means and standard deviations of local lick rates of all subjects over all sessions with the intermittent reinforcement schedule (FI $60 \mathrm{sec}$ ).
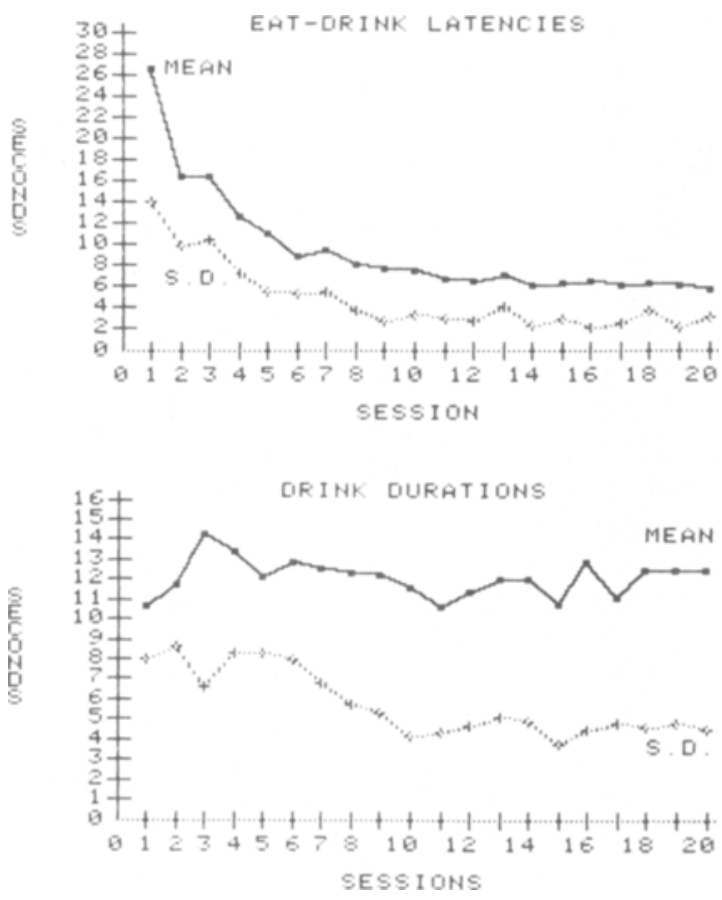

Figure 4. (Upper) Means and standard deviations of eat-drink latencies of all subjects over all sessions with the intermittent reinforcement schedule (FI $60 \mathrm{sec}$ ). (Lower) Means and standard deviations of individual drink durations of all subjects over all sessions with the intermittent reinforcement schedule (FI $60 \mathrm{sec}$ ). 
ence was significant $[t(7)=4.67, p<.01]$. No further changes in the standard deviation of drink-bout duration occurred after Session 12, and the ANOVA failed to reach significance over all 20 training days $[F(7,19)=1.57$, $p>.10]$.

The standard deviation of latencies from pellet delivery to the first subsequent lick also stabilized by Session 12 , although mean latencies continued a downward trend through to Session 20, as shown in the upper part of Figure 4. Latency decreases over sessions were statistically significant for both means $[F(7,19)=17.98$, $p<.0001]$ and standard deviations $[F(7,19)=9.16$, $p<.001]$, but neither local lick rates $[F(7,19)=0.51$, $p>.10]$ nor average drink-bout durations $[F(7,19)=$ $0.66, p>.10$ ] differed significantly when tested over all 20 training sessions. Thus, we found the development of adjunctive drinking to be characterized by increasing frequencies of postpellet drinks, decreasing eat-drink latencies, and tendencies to stereotypy in drink latency and duration, but not by differences in local lick rate from drink bouts taken under continuous reinforcement. Moreover, mean drink duration did not increase with training. Instead, it became less variable as drinking increased in frequency. The overall supernormal amount of drinking that characterizes schedule-induced polydipsia did not come about through bouts of licking that became longer or faster with training, but by way of drinks that became more frequent and more stereotyped in latency and duration.

\section{DISCUSSION}

Thirst-induced drinking by rats is regulated by physiological effects of water deficit (Rolls \& Rolls, 1982) and is characterized by lick rates of relatively little variability once drinking begins (Cone, 1974; Corbit \& Luschei, 1969; Hard, 1976; Keehn \& Arnold, 1960). Onset and duration times of individual drinks, however, apparently are random (Keehn, 1985; Premack, 1965); when and for how long a rat will take particular drinks in an unrestricted situation cannot yet be predetermined. But if feeding is constrained by a reinforcement or temporal schedule, occasions of drinks become highly predictable: most rats engage in an adjunctive drink bout almost every time pellet deliveries are interrupted (Keehn \& Colotla, 1971). Such drinks, we find, occur at about the same local rate of licking as drinks elicited by thirst, although by their frequency they lead to polydipsic levels of water intake that are not regulated by homeostatic mechanisms (Kissileff, 1973).

As we measured them, lap rates were somewhat lower than those reported elsewhere. These range from a low of 4.8 licks/sec reported by Hard (1976) to a high of 7.7 licks/sec reported by Corbit and Luschei (1969), depending on a particular animal and apparatus configuration. Thus, in a study by Hard (1976) with 3 rats for each distance, modal lick rates (measured as interlap times) declined linearly from 5.9 laps/sec when the drinking tube was $2 \mathrm{~mm}$ outside the cage wall to 5.0 laps/sec when this distance was $6 \mathrm{~mm}$. Two factors, at least, contribute to this variation. One is that occasional tube contacts other than licks, for example, pawing, sniffing, and biting, register as licks, and another is that contact with the animal's tongue may not break between licks, thus registering fewer licks than actually occur. Both these factors generate artificially low lick rates when they occur, and our choice of a minimum 3-sec burst of tube contacts in the definition of a lick bout may not have overcome this problem. However, this is less important in a comparative study such as ours than it is for the assessment of absolute lick-rate values. The point, nevertheless, needs clarification because it may relate to the vigor, or intensity, of individual licks, which Plonsky, Driscoll, Warren, and Rosellini (1984) imply is conditionable in the case of adjunctive drinking.

Several recent studies (e.g., Plonsky et al., 1985; Porter \& Hamm, 1984) trace the acquisition of polydipsia to Pavlovian conditioning of a motivational state to stimuli negatively associated with feeding. The increasing frequency and decreasing latency of adjunctive drinks that we found in the course of training is consistent with this interpretation. We did not, however, find an increase in magnitude of individual drinks, as measured by average lickbout durations. Instead, individual drink bouts became more stereotyped with training. Mean drink bout durations remained unchanged while the variability of bout durations significantly decreased. That is, whatever is conditioned in the acquisition of schedule-induced polydipsia, it is expressed by a mounting frequency of drinks that become stereotyped in latency and duration, but not by a change in the local rate of licking or in the average magnitude of drink durations. This finding bears on the relative contributions of operant and respondent conditioning to the development of the stimulus control of adjunctive drinking. Response magnitude is a traditional measure of respondent conditioning (Kimble, 1961; Rescorla \& Wagner, 1972) but not of operant conditioning. Response stereotypy, on the other hand, is characteristic of the development and maintenance of single operants (Antonitis, 1951; Herrick, 1965) or operant response sequences (Schwartz, 1982, 1986), but does not feature in the measurement of conditioned respondents. To this extent, our results point to some discriminative stimulus control of adjunctive drinking as an operant whether or not a Pavlovian conditioned motivational state is the impetus for adjunctive drinking.

Traditional theories of drinking have paid little attention to the details of licking behavior. They have focused on the origins of thirst sensations in water-deprived organisms. Modern theories are about determinants of drinking behavior; they include intracellular fluid depletion, reduction in extracellular volume, cholinergic and dopaminergic centers in the brain, and renin-angiotensin and histaminergic systems (see Fitzsimons, 1973, Kraly, 1984, and Rolls \& Rolls, 1982, for reviews). The significance of these mechanisms is typically assessed by the elicitation and blockage of drinking by suitable pharmacological or physiological agents, but, to our knowledge, 
there is no work on the effects of these agents on the timing of the drinking behavior that is elicited or blockedonly on the quantity of fluid consumed. For drinking elicited by intermittent reinforcement, at least, our work points to its powerful control over timing and to the possibility of distinctive mechanisms regulating the time of onset (latency) and the time of offset (duration) of drinking.

\section{REFERENCES}

ANTonitis, J. J. (1951). Response variability in the white rat during conditioning, extinction and reconditioning. Journal of Experimental Psychology, 42, 273-281.

Cone, D. M. (1974). Do mammals lick at a constant rate? A critical review of the literature. The Psychological Record, 24, 353-364.

Corbir, J. D., LuSCHEI, E. S. (1969). Invariance in the rat's rate of drinking. Journal of Comparative \& Physiological Psychology, 69, 119-125.

DAVIS, J. D. , KeEhN, J. D. (1959). Magnitude of reinforcement and consummatory behavior. Science, 130, 269-271.

FALK, J. L. (1961). Production of polydipsia in rats by an intermittent food schedule. Science, 133, 195-196.

FALK, J. L. (1972). The nature and determinants of adjunctive behavior. In R. M. Gilbert \& J. D. Keehn (Eds.), Schedule effects: Drugs, drinking and aggression (pp. 148-173). Toronto: University of Toronto Press.

FALK, J. L. (1981). The environmental generation of excessive behavior. In S. J. Mule (Ed.), Behavior in excess: An examination of the volitional disorders (pp. 313-337). New York: Free Press.

FITzsImons, J. T. (1973). Some historical perspectives in the physiology of thirst. In A. N. Epstein, H. R. Kissileff, \& E. Stellar (Eds.), The neuropsychology of thirst: New findings and advances in concepts (pp. 3-33). Washington, DC: Winston.

GiLBERT, T. F. (1958). Fundamental dimensional properties of the operant. Psychological Review, 65, 272-282.

$\mathrm{HARD}, \mathrm{E}$. (1976). The drinking pattern in the rat and its modulation through the effect of taste and previous experience (Alan Jacobs, Trans.). Stockholm: Esselte Studium.

Herrick, R. M. (1965). Level displacement under a fixed ratio schedule and subsequent extinction. Journal of Comparative \& Physiological Psychology, 59, 263-270.

KeEHN, J. D. (1985). Concurrent acquisition and maintenance of schedule-induced licking and biting by two strains of rats. The Psychological Record, 35, 559-574.

KeEHN, J. D., \& ARNOLD, E. M. M. (1960). Licking rates of albino rats. Science, 132, 739-741.

KeeHN, J. D., CoLotla, V. A. (1971). Stimulus and subject control of schedule-induced drinking. Journal of the Experimental Analysis of Behavior, 16, 257-262.

KIMBLE, G. A. (1961). Hilgard and Marquis' conditioning and learning. New York: Appleton-Century-Crofts.

KissilefF, H. R. (1973). Nonhomeostatic controls of drinking. In A.
N. Epstein, H. R. Kissileff, \& E. Stellar (Eds.), The neuropsychology of thirst: New findings and advances in concepts (pp. 163-198). Washington: Winston.

Kraly, F. S. (1984). Physiology of drinking elicited by eating. Psychological Review, 91, 478-490.

LOONEY, T. A., \& COHEN, P. S. (1982). Aggression induced by intermittent positive reinforcement. Neuroscience \& Biobehavioral Reviews, 6, 15-37.

Milienson, J. R., Allen, R., \& Pinker, S. (1977). Adjunctive drinking during variable and random interval food reinforcement schedules. Animal Learning \& Behavior, 5, 285-290.

Pear, J. J., Eldridge, G. D. (1984). The operant-respondent distinction: Future directions. Journal of the Experimental Analysis of Behavior, 42, 453-467.

Plonsky, M., Driscoll, C. D., a Roselinn, R. A. (1985). Polydipsia on fixed and variable time schedules as a function of signal condition in the rat. The Psychological Record, 35, 221-226.

Plonsky, M., Driscoll, C. D., WarRen, D. A., \& Rosellin, R. A. (1984). Do random time schedules induce polydipsia in the rat? Animal Learning \& Behavior, 12, 355-362.

Porter, J. H., \& Hamm, R. J. (1984). Associative control of scheduleinduced drinking. Animal Learning \& Behavior, 12, 339-340.

Premack, D. (1965). Reinforcement theory. In D. Levine (Ed.), Nebraska Symposium on Motivation (pp. 123-180). Lincoln: University of Nebraska Press.

Rescorla, R. A., \& Wagner, A. R. (1972). A theory of Pavlovian conditioning: Variations in the effectiveness of reinforcement and nonreinforcement. In A. H. Black \& W. F. Prokasy (Eds.), Classical conditioning II: Current research and theory. New York: AppletonCentury.

Rolss, B. J., \& Rolls, E. T. (1982). Thirst. Cambridge: Cambridge University Press.

ROPER, T. J. (1981). What is meant by the term "schedule-induced," and how general is schedule induction? Animal Learning \& Behavior, 9, 433-440.

SCHWARTZ, B. (1982). Failure to produce response variability with reinforcement. Journal of the Experimental Analysis of Behavior, 37, 171-182.

SCHWARTZ, B. (1986). Allocation of complex sequential operants on multiple and concurrent schedules of reinforcement. Journal of the Experimental Analysis of Behavior, 45, 283-295.

Segal, E. F., Oden, D. L., \& Deadwy ler, S. A. (1965). Determinants of polydipsia: IV. Free reinforcement schedule. Psychonomic Science, 3, 11-12.

Stellar, E., Hill, J. H. (1952). The rat's drinking rate as a function of water deprivation. Journal of Comparative \& Physiological Psychology, 45, 96-102.

TIMBerlake, W. (1982). Controls and schedule-induced behavior. Animal Learning \& Behavior, 10, 535-536.

WETHERINGTON, C. L. (1982). Is adjunctive behavior a third class of behavior? Neuroscience \& Biobehavioral Reviews, 6, 329-350.

(Manuscript received April 23, 1986; revision accepted for publication July 16, 1986.) 